PAPER • OPEN ACCESS

\title{
Quantisation of the elliptical Penning trap
}

To cite this article: Frances Crimin et al 2021 J. Phys. B: At. Mol. Opt. Phys. 54115501

View the article online for updates and enhancements.

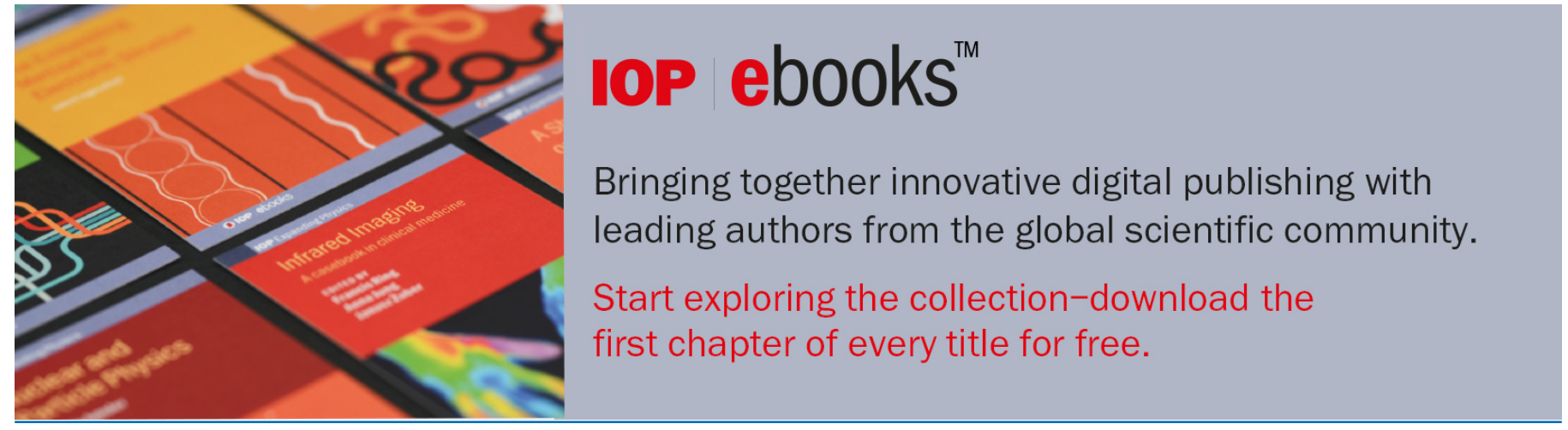

This content was downloaded from IP address 130.209 .6 .42 on $13 / 07 / 2021$ at $11: 43$ 


\title{
Quantisation of the elliptical Penning trap
}

\author{
Frances Crimin ${ }^{1,2, *}$, B , Barry M Garraway ${ }^{1}$ and José L Verdú ${ }^{1} \mathbb{C}$ \\ 1 Department of Physics and Astronomy, University of Sussex, Falmer BN1 9QH, United Kingdom \\ 2 Department of Physics and Astronomy, University of Glasgow, Glasgow G12 8QQ, United Kingdom \\ E-mail: frances.crimin@glasgow.ac.uk
}

Received 10 February 2021, revised 23 April 2021

Accepted for publication 7 May 2021

Published 9 June 2021

\begin{abstract}
We present the quantum theory of the elliptical Penning trap, i.e. the general case where the cylindrical symmetry of the electrostatic trapping potential around the trapping magnetic field axis is broken. The theory applies to both slightly and highly elliptical traps, where it is shown that the difference between the quantum states of particles in these traps corresponds to a variation of the degree of squeezing of their motional modes in the $x y$-plane. In a trap with tunable ellipticity, such as the Geonium Ghip planar Penning trap, it follows that control of the ellipticity via the trapping voltages enables squeezing of the quantum states of the particle. We discuss the adiabatic preparation of such squeezed states, which follows naturally from the appearance of an avoided crossing between the diabatic levels of the coupled motional states of the particle.
\end{abstract}

Keywords: Penning trap, quantum optics, elliptical Penning trap, trapped electrons

(Some figures may appear in colour only in the online journal)

\section{Introduction}

Since their initial development [1], Penning traps have become indispensable in high precision mass spectrometry $[2,3]$, in quantum electrodynamics (QED) measurements [4-6], and in anti-matter experiments [7-10]. The goal of achieving quantum information processing by confinement of single electrons in Penning traps [11-15] motivated the enterprise of planar traps [16-20], in an effort to optimise both the scalability of the system, and addressability of the trapped particles [21]. As an example, the Geonium Chip [22-25] belongs to the class of planar, elliptical Penning traps and is, furthermore, designed to form a compact, mobile device [26]. It is being developed for applications in quantum technology with trapped electrons, such as the detection and generation of quantum microwave radiation [27]. Inspired by the possibilities for enhanced control of the trapping potential in the Geonium Chip [25], this article develops the quantum description of charged parti-

* Author to whom any correspondence should be addressed.

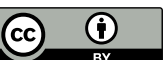

Original content from this work may be used under the terms of the Creative Commons Attribution 4.0 licence. Any further distribution of this work must maintain attribution to the author(s) and the title of the work, journal citation and DOI. cles in elliptical Penning traps, and discusses methods for the preparation of their states.

The theory of the elliptical Penning trap is discussed most notably by Kretzshmar [28], where it was shown that the classical Hamiltonian of the trap with a general quadrupolar electrostatic trapping potential without cylindric symmetry-as assumed in earlier work [29]—can be solved exactly by a proper canonical transformation. The rigorous classical treatment demonstrates how the trap's ellipticity parameter, $\epsilon$, can be used as a means of controlling the shape and frequency of the magnetron orbit of the particle. This is verified by numerical and experimental results in a trap with a tunable ellipticity [30]. The Geonium Chip also incorporates this function into its design [23], and it has been shown that the tunability of $\epsilon$ allows the dimensionality of this trap to be modified [25]. This paper aims to expand upon the theoretical work of [28] by analysing the quantum solution of the ideal elliptical Penning trap [31]. Following this, we show how the design of the Geonium Chip enables enhanced control of the quantum states of a single charged particle in the trap. While the results have general validity, we are mainly motivated by the use of trapped electrons, which can be driven into the quantum regime at cryogenic temperatures [32]. Alternatively, laser cooled ions [33] are also candidates for the novel manipulation of the quantum states presented here. 
This paper is divided into six sections. In section 2 , the ideal classical circular and elliptical traps are briefly reviewed, and the discussion extended to the design of the Geonium Chip trap. The quantum formalism is also introduced. The quantum solution of the elliptical trap is presented in section 3 , in the context of the well-known classical results [28]. The following section 4 reviews the Geonium Chip in more detail, and the experimental requirements for driving the trap to an 'ultraelliptical regime' [23] are discussed, with section 5 detailing the theory of this procedure. We summarise our results in section 6 .

\section{The ideal elliptical Penning trap}

\subsection{Classical theory}

2.1.1. The ideal classical circular Penning trap. In a radially (or cylindric) symmetric Penning trap, the ideal confining potential is the quadrupole

$$
\phi(\vec{r})=U_{0}\left(z^{2}-\frac{x^{2}+y^{2}}{2}\right),
$$

where the resulting static field is $\vec{E}=-\vec{\nabla} \phi(x, y, z)$ and the sign of $U_{0}$ depends upon the charge of the particles being trapped. Confinement in the radial direction is provided by a magnetic field $\vec{B}=|\vec{B}| \hat{e}_{z}$ with associated vector potential $\vec{A}(\vec{r})$, which we treat in the Coulomb gauge $\vec{\nabla} \cdot \vec{A}(\vec{r})=0$ such that $A_{x}=-|\vec{B}| y / 2$ and $A_{y}=|\vec{B}| x / 2$. The classical Hamiltonian of a particle of mass $m$ and charge $q$ in the presence of these crossed electromagnetic fields is given by [34]:

$$
\mathcal{H}=\frac{1}{2 m}(\vec{p}-q \vec{A}(\vec{r}))^{2}+q \phi(\vec{r}) .
$$

In this paper, the trapping of a single electron is considered, and inserting the explicit forms of $\vec{E}$ and $\vec{B}$ for $q<0$, (2) becomes

$$
\begin{aligned}
\mathcal{H}= & \frac{1}{2 m}\left(p_{x}^{2}+p_{y}^{2}+p_{z}^{2}\right)+\frac{\omega_{\mathrm{c}}}{2}\left(x p_{y}-y p_{x}\right) \\
& +\frac{1}{2} m\left(\frac{\omega_{1}}{2}\right)^{2}\left(x^{2}+y^{2}\right)+\frac{1}{2} m \omega_{z}^{2} z^{2},
\end{aligned}
$$

with definitions

$$
\omega_{\mathrm{c}}=\frac{|q \| \vec{B}|}{m}, \quad \omega_{z}=\sqrt{\frac{2 q U_{0}}{m}}, \quad \omega_{1}=\sqrt{\omega_{\mathrm{c}}^{2}-2 \omega_{z}^{2}},
$$

and where, as $q<0$, we also require $U_{0}<0$. The motion in the radial plane can be decoupled by canonical transformation [35], and the Hamiltonian written in terms of three distinct harmonic oscillators of the cyclotron $(+)$, axial $(z)$, and magnetron (-) modes. The magnetron motion is a result of the crossing of the electric and magnetic fields and contributes a negative energy in the Hamiltonian, and this instability must be minimised to prevent the electron striking the edge of the trap [36]. The frequencies of the decoupled modes are given explicitly by

$$
\omega_{+}=\frac{1}{2}\left(\omega_{\mathrm{c}}+\omega_{1}\right), \quad \omega_{-}=\frac{1}{2}\left(\omega_{\mathrm{c}}-\omega_{1}\right),
$$

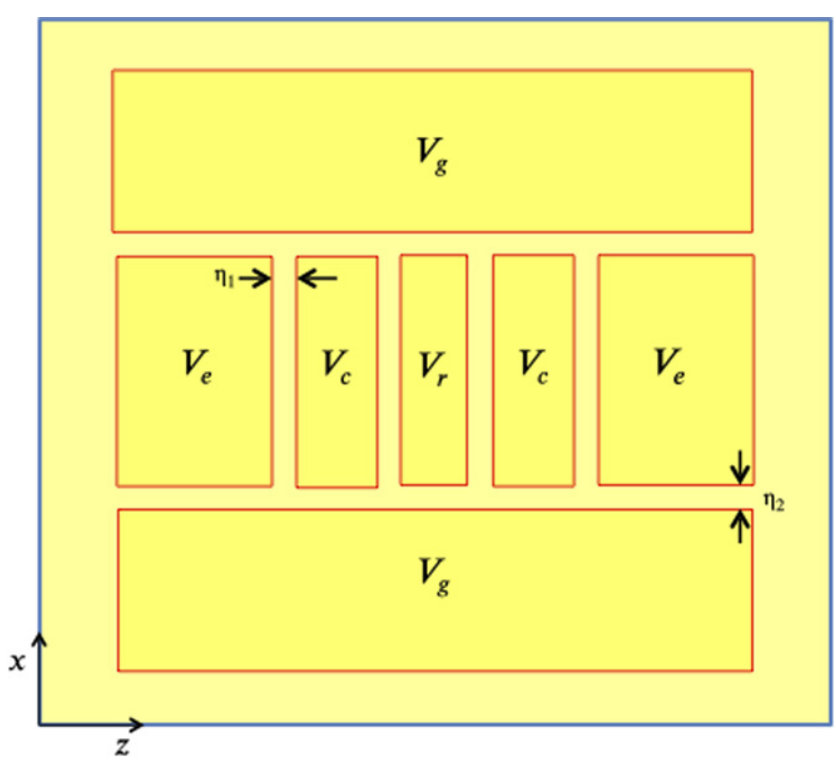

Figure 1. Electrode structure of the Geonium Chip, with $V_{\mathrm{r}}, V_{\mathrm{c}}, V_{\mathrm{e}}$, $V_{\mathrm{g}}$ indicating the ring, correction, endcap, and side-electrode voltages, respectively. Typical values of these voltages (in the $\epsilon \sim 0$ regime) are $V_{\mathrm{r}} \approx-1 \mathrm{~V}, V_{\mathrm{c}} \approx-1 \mathrm{~V}, V_{\mathrm{e}} \approx-2.7 \mathrm{~V}, V_{\mathrm{g}}=0 \mathrm{~V}$, with the length and width of the chip being $\sim 14 \mathrm{~mm}$ and $\sim 13 \mathrm{~mm}$ respectively. The equilibrium trapping position, $\left(0, y_{0}, 0\right)$, above the chip's surface is essentially determined by the voltage ratio $V_{\mathrm{c}} / V_{\mathrm{r}}$, and $V_{\mathrm{r}}$ further determines the overall scale of the trapping potential [25].

which comprise the rotational components of an epicyclic orbit in the radial plane of the trap. This is superposed upon harmonic motion with angular frequency $\omega_{z}$ along the $z$ axis.

\subsubsection{The Geonium Chip and the ideal classical elliptical Pen-} ning trap. As a concrete example of an elliptical Penning trap, but without loss of generality, here we consider the description of the Geonium Chip trap [22]. This trap essentially consists of the projection of a conventional cylindric trap [37] onto the surface of a chip. This results in the magnetic field being parallel to the surface of the chip. Its design aims to implement both the magnetic field source and the trap electrodes into a single chip, thus offering the potential of full scalability [23, 24]. A sketch of the trap is shown in figure 1 , where $V_{\mathrm{r}}, V_{\mathrm{c}}$, and $V_{\mathrm{e}}$ indicate the voltages applied to the ring, correction electrodes, and end-caps, respectively, and $V_{\mathrm{g}}$ denotes the voltage at the side-electrodes. It is these side-electrodes which are used to control the trap's ellipticity [25].

In figure 1 the gaps between the electrodes are labelled $\eta_{1}$ and $\eta_{2}$. These must be taken into account when engineering the total trapping potential $\Phi(\vec{r})$, calculated from the Green's function for the Laplace equation which fulfils Dirichlet's boundary conditions in a box [25]. The second order series expansion of this potential around the equilibrium position $\left(0, y_{0}, 0\right)$ is written in terms of coefficients $C_{i j k}$, defined

$$
C_{i j k}=\left.\frac{1}{i ! j ! k !} \cdot \frac{\partial^{i+j+k} \Phi(x, y, z)}{\partial x^{i} \partial y^{j} \partial z^{k}}\right|_{\left(0, y_{0}, 0\right)}
$$


so that the trapping potential $\Phi(\vec{r})$ is given by [22]:

$$
\begin{aligned}
\Phi(\vec{r})= & C_{002} \cdot\left(z^{2}-\frac{x^{2}+\left(y-y_{0}\right)^{2}}{2}\right) \\
& +\frac{1}{2} C_{002} \epsilon \cdot\left(x^{2}-\left(y-y_{0}\right)^{2}\right) .
\end{aligned}
$$

Here, the ellipticity parameter $\epsilon$ is defined as

$$
\epsilon=\frac{C_{200}-C_{020}}{C_{002}}
$$

and $-1<\epsilon<1$. In neglecting terms of higher order, we have assumed that the trap has been tuned sufficiently that these anharmonicities become vanishingly small [22]. Equation (7) represents the total potential supplied by the Geonium Chip, in the ideal elliptical potential approximation of a Penning trap [28]. Comparing this to (1), the axial frequency in the Geonium Chip is identified as

$$
\omega_{z}=\sqrt{\frac{2 C_{002} q}{m}}
$$

so that the Hamiltonian of this elliptical Penning trap can be written in terms of the radially symmetric Hamiltonian (3):

$$
\begin{aligned}
\mathcal{H}_{\epsilon}= & \mathcal{H}+\frac{1}{4} \epsilon m \omega_{z}^{2}\left(x^{2}-y^{2}\right) \\
= & \frac{1}{2 m}\left(p_{x}^{2}+p_{y}^{2}+p_{z}^{2}\right)+\frac{\omega_{\mathrm{c}}}{2}\left(x p_{y}-y p_{x}\right) \\
& +\frac{1}{2} m\left(\frac{\omega_{1}}{2}\right)^{2}\left(x^{2}+y^{2}\right)+\frac{1}{4} m \epsilon \omega_{z}^{2}\left(x^{2}-y^{2}\right)+\frac{1}{2} m \omega_{z}^{2} z^{2},
\end{aligned}
$$

where we draw particular attention to the contribution due to the ellipticity of the trap, by introducing a term which is proportional to $\epsilon$. The trapping height $y_{0}$ has been absorbed into the $y$ coordinate, such that $\left(y-y_{0}\right) \rightarrow y$, and will remain as such throughout this paper. This trapping height is primarily determined by the ratio $V_{\mathrm{c}} / V_{\mathrm{r}}$ in the example trap geometry [22]. The electrodes $V_{\mathrm{g}}$ enable variation of trap ellipticity [25], a feature further discussed in section 4 .

\subsection{Quantum theory}

2.2.1. The temperature of the Penning trap. The total energy in each of the three modes is determined by their respective temperatures $T_{+}, T_{-}$and $T_{z}$ via the cryogenic cooling of the system [29]. As the magnetron orbit is unstable and contributes a negative energy in the Hamiltonian, the temperature of the magnetron motion is also negative. The average quantum numbers of each, $\bar{n}_{\gamma}$, can be estimated from the simple energy equality

$$
\hbar \omega_{\gamma} \bar{n}_{\gamma}=k_{\mathrm{B}} T_{\gamma} ; \quad \gamma=+,-, z
$$

In the case of trapping an electron at cryogenic temperatures of $T_{+}=4.2 \mathrm{~K}$ or lower, and for sufficiently strong $\vec{B}$ fields, a quantum mechanical description of particle's cyclotron motion is required [29]. This is also true for the axial and magnetron motions when cooled by sideband-coupling [29] to the cyclotron motion. Treating the Hamiltonian of the trap as having three distinct harmonic contributions $\hat{H}_{+}, \hat{H}_{-}, \hat{H}_{z}$, the state of a particle in the system is described by the separable state

$$
\hat{\rho}_{+}\left(T_{+}\right) \otimes \hat{\rho}_{-}\left(T_{-}\right) \otimes \hat{\rho}_{z}\left(T_{z}\right)
$$

where [38]

$$
\hat{\rho}_{\gamma}=\frac{\exp \left(-\beta_{\gamma} \hat{H}_{\gamma}\right)}{\operatorname{Tr}\left[\exp \left(-\beta_{\gamma} \hat{H}_{\gamma}\right)\right]} ; \quad \beta_{\gamma}=\left(k_{\mathrm{B}} T_{\gamma}\right)^{-1}, \quad \gamma=+,-, z
$$

For the following discussion, it is assumed that the trap is held at sufficiently low temperatures that only the lowest states $(n \lesssim 10)$ are occupied.

2.2.2. Canonical quantisation. Conventional treatment in Penning trap theory $[28,29,35,39]$ follows a procedure of classical canonical transformation followed by quantisation in order to diagonalise the Hamiltonian of the radially symmetric trap (3). In the present work, we first quantize the Hamiltonian, and introduce creation and annihilation operators corresponding to motion along the $x, y$ and $z$ axes:

$$
\begin{aligned}
& \hat{a}_{x}=\frac{1}{\sqrt{2 \hbar}}\left(\sqrt{\frac{m \omega_{1}}{2}} \hat{x}+\mathrm{i} \sqrt{\frac{2}{m \omega_{1}}} \hat{p}_{x}\right), \\
& \hat{a}_{x}^{\dagger}=\frac{1}{\sqrt{2 \hbar}}\left(\sqrt{\frac{m \omega_{1}}{2}} \hat{x}-\mathrm{i} \sqrt{\frac{2}{m \omega_{1}}} \hat{p}_{x}\right), \\
& \hat{a}_{y}=\frac{1}{\sqrt{2 \hbar}}\left(\sqrt{\frac{m \omega_{1}}{2}} \hat{y}+\mathrm{i} \sqrt{\frac{2}{m \omega_{1}}} \hat{p}_{y}\right), \\
& \hat{a}_{y}^{\dagger}=\frac{1}{\sqrt{2 \hbar}}\left(\sqrt{\frac{m \omega_{1}}{2}} \hat{y}-\mathrm{i} \sqrt{\frac{2}{m \omega_{1}}} \hat{p}_{y}\right), \\
& \hat{a}_{z}=\frac{1}{\sqrt{2 \hbar}}\left(\sqrt{\frac{m \omega_{z}}{2}} \hat{z}+\mathrm{i} \sqrt{\frac{2}{m \omega_{z}}} \hat{p}_{z}\right), \\
& \hat{a}_{z}^{\dagger}=\frac{1}{\sqrt{2 \hbar}}\left(\sqrt{\frac{m \omega_{z}}{2}} \hat{z}-\mathrm{i} \sqrt{\frac{2}{m \omega_{z}}} \hat{p}_{z}\right),
\end{aligned}
$$

which obey commutation relations: $\left[\hat{a}_{x}, \hat{a}_{x}^{\dagger}\right]=\left[\hat{a}_{y}, \hat{a}_{y}^{\dagger}\right]=$ $\left[\hat{a}_{z}, \hat{a}_{z}^{\dagger}\right]=1$, with all other commutators being zero. The quantum Hamiltonian is non-diagonal in this basis, so we define the raising and lowering operators of the cyclotron and magnetron modes:

$$
\begin{array}{ll}
\hat{a}_{+}=\frac{1}{\sqrt{2}}\left(\hat{a}_{x}-\mathrm{i} \hat{a}_{y}\right), & \hat{a}_{+}^{\dagger}=\frac{1}{\sqrt{2}}\left(\hat{a}_{x}^{\dagger}+\mathrm{i} \hat{a}_{y}^{\dagger}\right), \\
\hat{a}_{-}=\frac{1}{\sqrt{2}}\left(\hat{a}_{x}+\mathrm{i} \hat{a}_{y}\right), & \hat{a}_{-}^{\dagger}=\frac{1}{\sqrt{2}}\left(\hat{a}_{x}^{\dagger}-\mathrm{i} \hat{a}_{y}^{\dagger}\right),
\end{array}
$$

which commute appropriately due to the unitary nature of the transformation (17). We now write the Hamiltonian of the 
radially symmetric trap in the standard way:

$\hat{\mathcal{H}}_{0}=\hbar \frac{\omega_{+}}{2}\left(\hat{n}_{+}+\frac{1}{2}\right)-\hbar \frac{\omega_{-}}{2}\left(\hat{n}_{-}+\frac{1}{2}\right)+\hbar \omega_{z}\left(\hat{n}_{z}+\frac{1}{2}\right)$,

where $\hat{n}_{\gamma}=\hat{a}_{\gamma}^{\dagger} \hat{a}_{\gamma}$; such notation is adopted throughout the remainder of this article.

The additional elliptical contribution $(\propto \epsilon)$ in equation (10) can be written directly in terms of these mode operators, so that the Hamiltonian of the ideal elliptical Penning trap is given by:

$$
\begin{aligned}
\hat{\mathcal{H}}_{\epsilon}= & \hbar \omega_{+}\left(\hat{n}_{+}+\frac{1}{2}\right)-\hbar \omega_{-}\left(\hat{n}_{-}+\frac{1}{2}\right)+\hbar \omega_{z}\left(\hat{n}_{z}+\frac{1}{2}\right) \\
& +\frac{1}{4} m \epsilon \omega_{z}^{2}\left(\frac { \hbar } { m \omega _ { 1 } } \left(\hat{a}_{+}^{\dagger} \hat{a}_{+}^{\dagger}+\hat{a}_{+} \hat{a}_{+}\right.\right. \\
& \left.\left.-\left(\hat{a}_{-}^{\dagger} \hat{a}_{-}^{\dagger}+\hat{a}_{-} \hat{a}_{-}\right)+2 \mathrm{i}\left(\hat{a}_{+}^{\dagger} \hat{a}_{-}-\hat{a}_{-}^{\dagger} \hat{a}_{+}\right)\right)\right) .
\end{aligned}
$$

\section{Solving the quantum elliptical Hamiltonian}

\subsection{Two-mode unitary transformation}

We diagonalise Hamiltonian (19) by proceeding in two parts, and begin by defining a unitary transformation operator $\hat{U}$ [40]:

$$
\hat{U}=\exp \left\{\mathrm{i} \frac{\theta}{2}\left(\hat{a}_{+}^{\dagger} \hat{a}_{-}+\hat{a}_{-}^{\dagger} \hat{a}_{+}\right)\right\} .
$$

The transformation operator (20) is applied to $\hat{\mathcal{H}}_{\epsilon}$, and setting

$$
\theta=\arctan (\gamma) ; \quad \gamma=\frac{\epsilon \omega_{z}^{2}}{\omega_{1} \omega_{\mathrm{c}}},
$$

produces

$$
\begin{aligned}
\hat{\mathcal{H}}_{\epsilon}^{\prime}= & \frac{\hbar \omega_{1}}{2}\left(\hat{n}_{+}+\hat{n}_{-}+1\right)+\frac{\hbar \omega_{\mathrm{c}} \sqrt{1+\gamma^{2}}}{2}\left(\hat{n}_{+}-\hat{n}_{-}\right) \\
& +\hbar \omega_{z}\left(\hat{n}_{z}+\frac{1}{2}\right) \\
& +\frac{\hbar}{4} \frac{\epsilon \omega_{z}^{2}}{\omega_{1}}\left(\hat{a}_{+}^{\dagger} \hat{a}_{+}^{\dagger}+\hat{a}_{+} \hat{a}_{+}-\hat{a}_{-}^{\dagger} \hat{a}_{-}^{\dagger}-\hat{a}_{-} \hat{a}_{-}\right)
\end{aligned}
$$

\subsection{Squeezing the Hamiltonian}

The remaining non-diagonal terms of (22), i.e. those proportional to $\epsilon$, can be written in diagonal form by application of squeezing operators $\hat{S}\left(\zeta_{+}\right)$and $\hat{S}\left(\zeta_{-}\right)$, the general form of which is given by $[38]^{3}$ :

$$
\begin{array}{ll}
\hat{S}\left(\zeta_{+}\right)=\exp \left\{-\frac{\zeta_{+}}{2} \hat{a}_{+}^{\dagger 2}+\frac{\zeta_{+}^{*}}{2} \hat{a}_{+}^{2}\right\} ; & \zeta_{+}=r_{+} \exp \left(\mathrm{i} \phi_{+}\right), \\
\hat{S}\left(\zeta_{-}\right)=\exp \left\{-\frac{\zeta_{-}}{2} \hat{a}_{-}^{\dagger 2}+\frac{\zeta_{-}^{*}}{2} \hat{a}_{-}^{2}\right\} ; & \zeta_{-}=r_{-} \exp \left(\mathrm{i} \phi_{-}\right),
\end{array}
$$

\footnotetext{
${ }^{3}$ The notation here has been chosen in an effort to reduce clutter in calculations. The arguments of $\hat{S}\left(\zeta_{+}\right)$and $\hat{S}\left(\zeta_{-}\right)$additionally indicate the Hilbert space in which they operate; the notation is shorthand for $\hat{S}_{+}\left(\zeta_{+}\right), \hat{S}_{-}\left(\zeta_{-}\right)$.
}

where $r_{+}, r_{-} \in \mathfrak{R}$. These act upon their appropriate operators in the following way [38]:

$$
\begin{aligned}
\hat{S}\left(\zeta_{\tau}\right) \hat{a}_{\tau} \hat{S}^{\dagger}\left(\zeta_{\tau}\right)= & \hat{a}_{\tau} \cosh \left(r_{\tau}\right) \\
& +\hat{a}_{\tau}^{\dagger} \sinh \left(r_{\tau}\right) \exp \left(\mathrm{i} \phi_{\tau}\right) ; \quad \tau=+,-
\end{aligned}
$$

Following the classical solution in [28], we introduce the definitions

$$
\kappa=\frac{\epsilon \omega_{z}^{2}}{2 \omega_{1}}, \quad K(\kappa)=\frac{\omega_{\mathrm{c}}}{2 \kappa}\left[\sqrt{1+\frac{4 \kappa^{2}}{\omega_{\mathrm{c}}^{2}}}-1\right],
$$

and choose the real-valued squeezing parameters

$$
\begin{array}{rlrl}
\zeta_{+} & =\frac{1}{2} \tanh ^{-1}\left(\gamma_{+}\right) ; & \gamma_{+} & =-\frac{\kappa}{\omega_{+}+\kappa K(\kappa)}, \\
\zeta_{-} & =\frac{1}{2} \tanh ^{-1}\left(\gamma_{y}\right) ; & \gamma_{y}=-\frac{\kappa}{\omega_{-}+\kappa K(\kappa)},
\end{array}
$$

such that $\phi_{+}=\phi_{-}=0$. Acting $\hat{S}\left(\zeta_{+}\right)$and $\hat{S}\left(\zeta_{-}\right)$in (23) upon Hamiltonian (22) produces [31]

$$
\begin{aligned}
\hat{\mathcal{H}}_{\zeta} & =\hat{S}\left(\zeta_{+}\right) \hat{S}\left(\zeta_{-}\right) \hat{\mathcal{H}}_{\epsilon}^{\prime} \hat{S}^{\dagger}\left(\zeta_{+}\right) \hat{S}^{\dagger}\left(\zeta_{-}\right) \\
& =\hbar \tilde{\omega}_{+}\left(\hat{n}_{+}+\frac{1}{2}\right)-\hbar \tilde{\omega}_{-}\left(\hat{n}_{-}+\frac{1}{2}\right)+\hbar \omega_{z}\left(\hat{n}_{z}+\frac{1}{2}\right),
\end{aligned}
$$

where [28]

$$
\begin{aligned}
& \tilde{\omega}_{+}=\sqrt{\omega_{+}^{2}+\omega_{1} \kappa K(\kappa)}=\sqrt{\frac{1}{2}\left(\omega_{\mathrm{c}}^{2}-\omega_{z}^{2}\right)+\frac{1}{2} \sqrt{\omega_{\mathrm{c}}^{2} \omega_{1}^{2}+\epsilon^{2} \omega_{z}^{4}}}, \\
& \tilde{\omega}_{-}=\sqrt{\omega_{-}^{2}-\omega_{1} \kappa K(\kappa)}=\sqrt{\frac{1}{2}\left(\omega_{\mathrm{c}}^{2}-\omega_{z}^{2}\right)-\frac{1}{2} \sqrt{\omega_{\mathrm{c}}^{2} \omega_{1}^{2}+\epsilon^{2} \omega_{z}^{4}}} .
\end{aligned}
$$

This result corresponds to the classical solution [28], and it is indeed what we would expect from other treatment of the elliptical trap [20, 41]. However, here we have shown the ultimate dependence of the squeezing parameters upon the trap voltages, through the dependence of the squeezing parameters on $\epsilon$ in (25) and (26), and the dependence of $\epsilon$ on the expansion coefficients of the trapping potential (8). This explicit dependence is the central result of this paper, allowing for the possibility of preparing squeezed quantum states of a Penning trap held at low temperatures. As discussed in [28], the frequency of the cyclotron motion is little affected by a changing ellipticity parameter, whereas the magnetron frequency decreases rapidly as $|\epsilon| \rightarrow 1$. The mode frequencies (28) reduce to $\omega_{+}$ and $\omega_{-}$in (5) as $\epsilon \rightarrow 0$. The eigenstates of the system can be found by applying $\hat{U}^{\dagger} \hat{S}^{\dagger}\left(\zeta_{+}\right) \hat{S}^{\dagger}\left(\zeta_{-}\right)$to solutions of the diagonal Hamiltonian (27). One such solution is given by coherent states, and it is straightforward to show that semiclassical solutions of the trap lead to equations of motion again in agreement with those found from classical analysis [28]. 


\section{Tuning the ellipticity of the Penning trap: classical analysis}

The results so far hold for any elliptical Penning trap, and reduce to those for circular traps when $\epsilon=0$ [40]. This section considers a more specific example of where we can exploit the dependence of the squeezing parameters on the trap voltages: variation of the ellipticity parameter in the Geonium Chip trap. A more complete discussion of this can be found in [25].

\subsection{The ground planes $V_{\mathrm{g}}$ in the Geonium Chip}

As mentioned in section 2.2, the five electrodes in the chip derived from the conventional cylindrical trap are flanked by two additional side-electrodes, held at voltages $V_{\mathrm{g}}$. The typical hierarchy of voltages used for trapping is $V_{\mathrm{e}}>V_{\mathrm{c}} \simeq V_{\mathrm{r}}$, and $V_{\mathrm{g}}=0$. This section briefly examines the operation of the Geonium Chip when the side-electrodes are held at finite voltage.

A finite voltage $V_{\mathrm{g}}$ affects the coefficients $C_{\mathrm{ijk}}(6)$ in the series expansion of the trapping potential $\Phi(\vec{r})$. According to equation (8), this modifies the ellipticity parameter $\epsilon$ of the trap, which can be tuned to any particular value $-1<\epsilon<1$ [25]. In turn, this alters the mode frequencies (28) and orbits of the electron, which are given by [28]

$$
\begin{aligned}
& x(t)=\xi_{+} \cdot \tilde{A}_{+} \cos \left(\tilde{\omega}_{+} t\right)+\xi_{-} \cdot \tilde{A}_{-} \cos \left(\tilde{\omega}_{-} t\right), \\
& y(t)=-\eta_{+} \cdot \tilde{A}_{+} \sin \left(\tilde{\omega}_{+} t\right)-\eta_{-} \cdot \tilde{A}_{-} \sin \left(\tilde{\omega}_{-} t\right),
\end{aligned}
$$

where

$$
\begin{aligned}
& \xi_{ \pm}=\sqrt{\frac{\omega_{\mathrm{c}}^{2}+\epsilon \omega_{z}^{2} \pm \sqrt{\omega_{\mathrm{c}}^{2} \omega_{1}^{2}+\epsilon^{2} \omega_{z}^{4}}}{2 \tilde{\omega}_{ \pm} / \omega_{1} \sqrt{\omega_{\mathrm{c}}^{2} \omega_{1}^{2}+\epsilon^{2} \omega_{z}^{4}}}}, \\
& \eta_{ \pm}=\sqrt{\frac{\omega_{\mathrm{c}}^{2}-\epsilon \omega_{z}^{2} \pm \sqrt{\omega_{\mathrm{c}}^{2} \omega_{1}^{2}+\epsilon^{2} \omega_{z}^{4}}}{2 \tilde{\omega}_{ \pm} / \omega_{1} \sqrt{\omega_{\mathrm{c}}^{2} \omega_{1}^{2}+\epsilon^{2} \omega_{z}^{4}}}}
\end{aligned}
$$

and [22]:

$$
\tilde{A}_{+}=\sqrt{\frac{2 E_{+}}{m\left(\tilde{\omega}_{+}^{2}-\frac{\omega_{z}^{2}}{2}\right)}}, \quad \tilde{A}_{-}=\sqrt{\frac{2 E_{-}}{m\left(\tilde{\omega}_{-}^{2}-\frac{\omega_{z}^{2}}{2}\right)}} .
$$

$E_{+}$and $E_{-}$denote the cyclotron and magnetron energies, respectively.

The cyclotron frequency, typically in the $\mathrm{GHz}$ range, along with the coefficients $\xi_{+}$and $\eta_{+}$in (30), are largely unaffected by the ellipticity: $\left|\xi_{+}\right| \simeq\left|\eta_{+}\right| \simeq 1$ [28]. In contrast, the magnetron motion, through the frequency $\tilde{\omega}_{-}$and coefficients $\xi_{-}, \eta_{-}$, is significantly modified by a non-zero voltage $V_{\mathrm{g}}$. As $\epsilon$ increases, the magnetron motion becomes a narrow ellipse along the $x$ axis, so that in the limit $\epsilon \rightarrow 1$, the magnetron orbit is confined to this axis. This is discussed more extensively in [25]. The cyclotron orbit is a fast oscillation superposed on top of the magnetron motion with $\tilde{A}_{+} \ll \tilde{A}_{-}$. Including the axial oscillation, the motion of the electron therefore becomes quasi two-dimensional (2D) as $\epsilon \rightarrow 1$. This is known as the 'ultra-elliptical regime' of the Penning trap [25].

\subsection{The ultra-elliptical regime}

The ultra-elliptical regime is reached by starting with the trapped electron in the regime where $|\epsilon| \lesssim 0.9$. As discussed in [25], the semi-major axis of the magnetron ellipse becomes increasingly large with $\epsilon$. Thus, in order that the electron does not orbit outside the harmonic trapping region while driving to the $\epsilon \rightarrow 1$ regime, magnetron-sideband cooling must be applied between successive small increments of the sideelectrode voltage, $V_{\mathrm{g}}$. The former technique reduces the average quantum number of the magnetron motion $\bar{n}_{-}$until it equals that of the axial mode [29], which accordingly reduces the energy of this mode and the size of the amplitude $\tilde{A}_{-}$.

The series expansion of the potential $\Phi(\vec{r})$ is around the equilibrium position $\left(0, y_{0}, 0\right)$, where $y_{0}$ itself is determined by the equation $\partial_{y} \Phi(0, y, 0)=0$. As the $C_{i j k}$ coefficients in this expansion change with $V_{\mathrm{g}}$, in turn anharmonicities of order $3(i+j+k=3)$ and $4(i+j+k=4)$ are generated with each new voltage of the ground plane [25]. These anharmonicities generate an undesired linear dependence of the motional frequencies with the particle's energies [22]. In order to compensate for this, the correction-electrode voltage $V_{\mathrm{c}}$ must be adapted to a new optimal value $V_{\mathrm{c}}^{\mathrm{opt}}$ for each new value of the ground plane, and hence each new $y_{0}$, so that the linear dependence of $\omega_{z}$ with the axial energy is eliminated. The precise determination of all the frequencies of the trapped particle critically relies on $V_{c}^{\text {opt }}$ being well defined [22, 29].

In summary, increasing the ellipticity in the Geonium Chip through $V_{\mathrm{g}}$ changes $y_{0}$, and in turn the anharmonic coefficients in $\Phi(\vec{r})(7)$. These are accordingly eliminated by modifying $V_{\mathrm{c}}$ to some value $V_{\mathrm{c}}^{\mathrm{opt}}$. The relationship between the required $V_{\mathrm{c}}^{\mathrm{opt}}$ with increasing $V_{\mathrm{g}}$ is extensively discussed in [25]. A particular relationship between these values must be maintained in order to achieve experimental adiabaticity. This leads to welldefined frequencies $\tilde{\omega}_{z}$ and $\tilde{\omega}_{-}$, enabling magnetron cooling to be applied at every stage of the ramping process as the trap is driven towards the ultra-elliptical regime [25].

\section{Tuning the ellipticity of the Penning trap: quantum analysis}

This section contains theoretical analysis of the experimental procedure described in section 4.2 for achieving the regime $\epsilon \rightarrow 1$. Beginning in section 5.1 with the process of increasing the ellipticity, we then go on to discuss the process of cooling the magnetron motion in section 5.2.

\subsection{Squeezing the axes}

As discussed in section 4.2, the ellipticity must be varied stepwise such that an optimal tuning ratio, $V_{\mathrm{c}}^{\mathrm{opt}}$, is maintained at every increment of $\epsilon$. Denoting the total ellipticity of the trap $\epsilon_{k}$, and each variation $\delta \epsilon_{k}$, then the ellipticity after each step is given by

$$
\epsilon_{k+1}=\epsilon_{k}+\delta \epsilon_{k}
$$



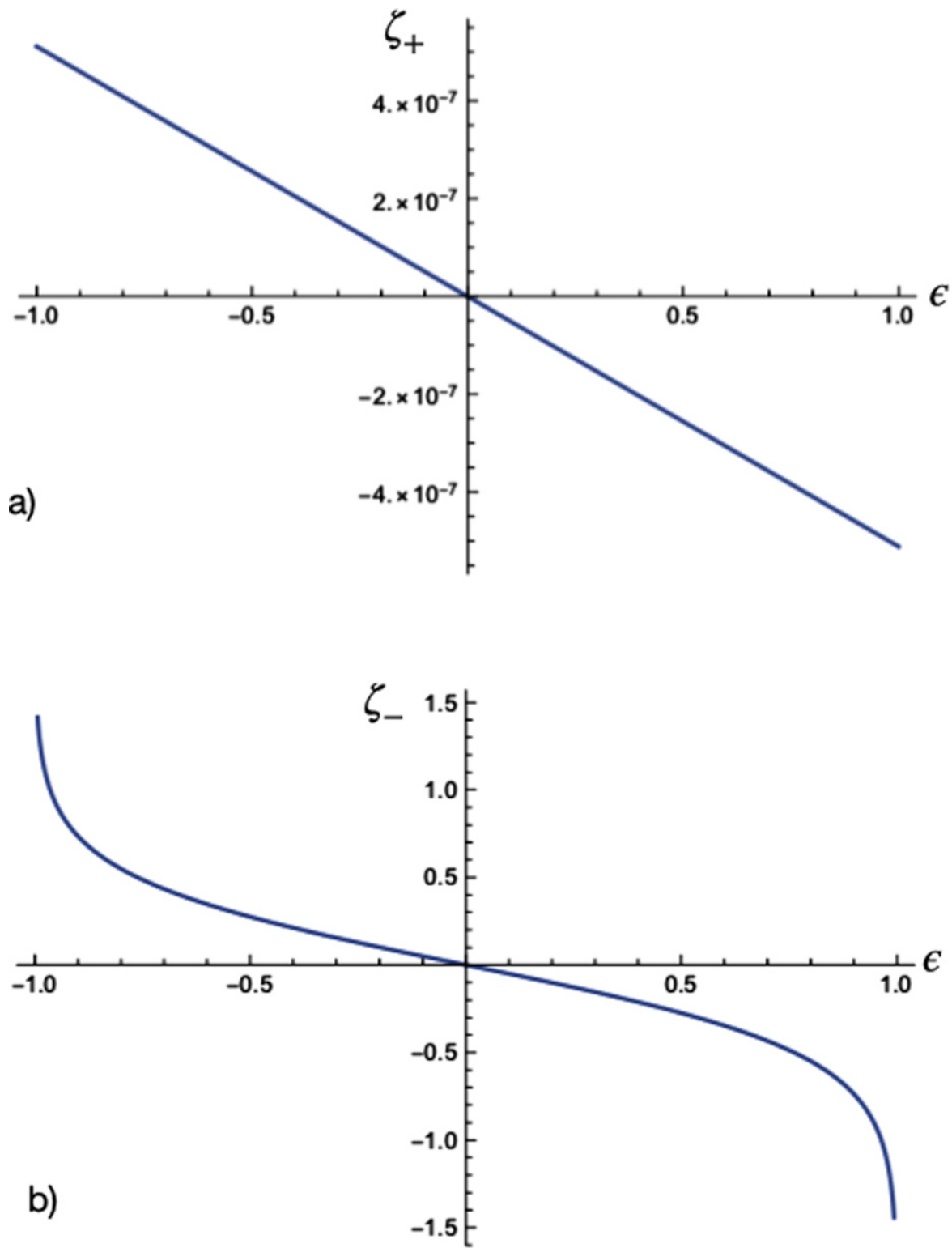

Figure 2. The squeezing parameters a) $\zeta_{+}(\epsilon)$ and b) $\zeta_{-}(\epsilon)$ as given in equation (33) with $\epsilon=\delta \epsilon_{k}$ and $\epsilon_{k}=0$. We have plotted values for trap parameters $|\vec{B}|=0.5 \mathrm{~T}$ and $V_{\mathrm{r}}=-1 \mathrm{~V}$, so that $\omega_{z} \approx 125 \mathrm{MHz}$, and $\omega_{\mathrm{c}}(0) \approx \omega_{1}(0) \approx 90 \mathrm{GHz}$. Comparing the scale of the axis on each plot, it is clear that the $x$ (cyclotron) squeezing parameter is little affected by a changing value of $\epsilon$, whereas that of the $y$ (magnetron) motion varies significantly.

At each increase $\delta \epsilon_{k}$ of the ellipticity $\epsilon_{k}$, the solutions of the trap change, with the squeezing parameters $\zeta_{+}$and $\zeta_{-}$now dependent on both $\delta \epsilon_{k}$ and $\epsilon_{k}$ :

$$
\begin{aligned}
& \zeta_{+}\left(\epsilon_{k}, \delta \epsilon_{k}\right)=\tanh ^{-1}\left(\frac{-\delta \epsilon_{k} \omega_{z}^{2} / 2 \tilde{\omega}_{1}\left(\epsilon_{k}\right)}{\tilde{\omega}_{+}\left(\epsilon_{k}\right)+\sqrt{\left(\frac{\tilde{\omega}_{\mathrm{c}}\left(\epsilon_{k}\right)}{2}\right)^{2}+\left(\frac{\delta \epsilon_{\epsilon_{k}} \omega_{z}}{2 \tilde{\omega}_{1}\left(\epsilon_{k}\right)}\right)^{2}}-\frac{\tilde{\omega}_{\mathrm{c}}\left(\epsilon_{k}\right)}{2}}\right), \\
& \zeta_{-}\left(\epsilon_{k}, \delta \epsilon_{k}\right)=\tanh ^{-1}\left(\frac{-\delta \epsilon_{k} \omega_{z}^{2} / 2 \tilde{\omega}_{1}\left(\epsilon_{k}\right)}{\tilde{\omega}_{-}\left(\epsilon_{k}\right)+\sqrt{\left(\frac{\tilde{\omega}_{\mathrm{c}}\left(\epsilon_{k}\right)}{2}\right)^{2}+\left(\frac{\delta \epsilon_{k} \omega_{z}}{2 \tilde{\omega}_{1}\left(\epsilon_{k}\right)}\right)^{2}}-\frac{\tilde{\omega}_{\mathrm{c}}\left(\epsilon_{k}\right)}{2}}\right),
\end{aligned}
$$

where $\tilde{\omega}_{\mathrm{c}}$ and $\tilde{\omega}_{1}$ are given in (A.2). The rotation angle $\theta(21)$ is also modified, see (A.1). Figure 2 shows the squeezing param- eters $\zeta_{+}$and $\zeta_{-}$from equation (33), with $\epsilon_{k}=\epsilon_{0}=0$, so that $\delta \epsilon_{k}=\epsilon$ is the total ellipticity, as plotted along the $x$ axis. The plots illustrate how the physical orbits of the cyclotron and magnetron motions are so differently affected by the increasing ellipticity: the squeezing parameter $\zeta_{+}$varies very little with $\epsilon$, whereas $\zeta_{-}$tends to $\pm \infty$ as $\epsilon \rightarrow \pm 1$. Thus, the cyclotron orbit is largely unaffected by the ellipticity, whereas the magnetron motion is squeezed to a line along the $x(y)$ axis when $\epsilon \rightarrow 1(\epsilon \rightarrow-1)$.

\subsection{Cooling the magnetron motion}

Sideband-coupling of the axial and magnetron modes is now considered. As mentioned in section 4.2, this is required for preventing the magnetron orbit from becoming too large 
Bare and dressed energy of the combined axial and

magnetron spectrum for the $N=1$ levels

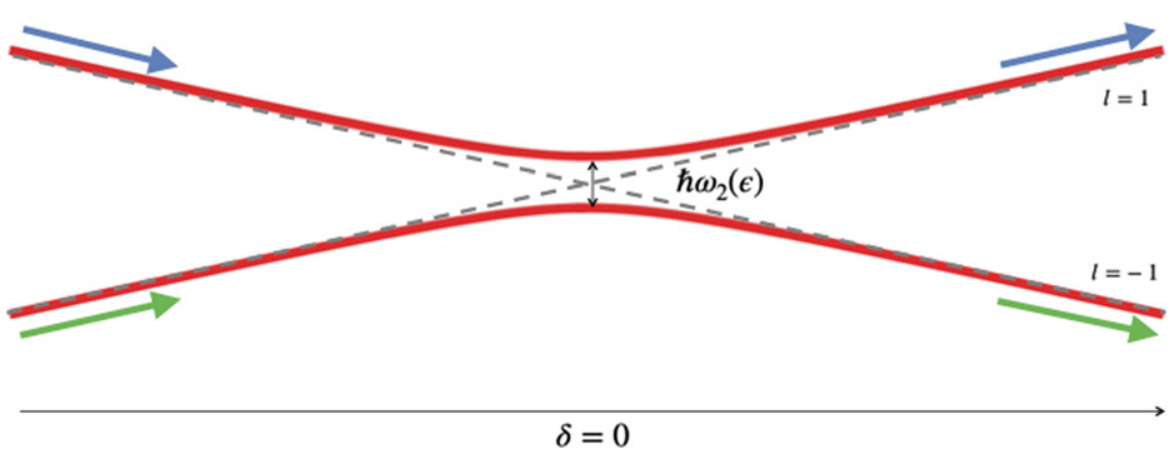

Figure 3. An avoided crossing between the $n_{-}=1, n_{z}=0$ (upper) and $n_{-}=0, n_{z}=1$ (lower) levels can be used to discuss quantum adiabaticity requirements for the axial-magnetron coupling stages of driving to the ultra-elliptical regime [25, 31]. A large enough gap between the dressed levels (or an instantaneous switching on of the coupling field) means that the system either follows the blue or green paths as the gap at $\delta=0$ is created, so that the electron remains in an adiabatic state.

when driving the trapping potential towards the ultra-elliptical regime. A more detailed calculation of the process is presented in appendix B, with the main results quoted below.

Coupling of the motional modes in the elliptical Penning trap can be achieved by a time-dependent electric field [42]

$$
\vec{E}\left(\epsilon_{k}, t\right)=\operatorname{Re}\left(\xi \mathrm{e}^{\mathrm{i} \tilde{\omega}_{m}\left(\epsilon_{k}\right) t}\right)\left(x \hat{e}_{z}+z \hat{e}_{x}\right),
$$

where we have included the ellipticity $\epsilon_{k}$ as a reminder that the frequency of field will vary with each increment $\delta \epsilon_{k}$. Such a field (with $\xi \in \mathfrak{R}$ ) has an associated potential

$$
V\left(x, z, \epsilon_{k}, t\right)=-\xi \cos \left(\tilde{\omega}_{m}\left(\epsilon_{k}\right) t\right)(x z) .
$$

After quantisation and transformation by $\hat{U}, \hat{S}\left(\zeta_{+}\right)$and $\hat{S}\left(\zeta_{-}\right)$, the explicit time dependence of the potential can be removed by transformation to a rotating frame, resulting in the total Hamiltonian of the system

$$
\begin{aligned}
\hat{\mathcal{H}}_{\zeta t}= & \omega_{0}\left(\epsilon_{k}\right) \hat{K}_{0}+\delta \hat{K}_{3}-\omega_{2}\left(\epsilon_{k}\right) \hat{K}_{2} \\
& +\frac{\hbar \omega_{0}\left(\epsilon_{k}\right)}{2}+\hbar \tilde{\omega}_{+}\left(\hat{n}_{+}+\frac{1}{2}\right),
\end{aligned}
$$

where [31]

$$
\begin{aligned}
\hat{K}_{0} & =\frac{\hbar}{2}\left(\hat{n}_{-}+\hat{n}_{z}\right), \\
\hat{K}_{1} & =\frac{\hbar}{2}\left(\hat{a}_{-}^{\dagger} \hat{a}_{z}+\hat{a}_{z}^{\dagger} \hat{a}_{-}\right), \\
\hat{K}_{2} & =-\frac{i \hbar}{2}\left(\hat{a}_{-}^{\dagger} \hat{a}_{z}-\hat{a}_{z}^{\dagger} \hat{a}_{-}\right), \\
\hat{K}_{3} & =\frac{\hbar}{2}\left(\hat{n}_{-}-\hat{n}_{z}\right),
\end{aligned}
$$

and $\omega_{0}\left(\epsilon_{k}\right), \omega_{2}\left(\epsilon_{k}\right)$ are given in (B.10). These are the Schwinger boson angular momentum vectors of the 2D harmonic oscillator system comprised of the magnetron and axial mode operators of the trap, obeying appropriate commutation relations [43]

$$
\left[\hat{K}_{i}, \hat{K}_{j}\right]=i \hbar \varepsilon_{i j k} \hat{K}_{k}, \quad\left[\hat{K}_{0}, \hat{K}_{i}\right]=0 ; \quad i=1,2,3 .
$$

The eigenstates and eigenvalues of $\hat{K}_{0}$ and $\hat{K}_{3}$ are straightforward, and the quantum numbers $N=n_{-}+n_{z}, l=n_{-}-n_{z}$ defined so that [43]:

$$
\begin{aligned}
\hat{K}_{0}\left|n_{-}, n_{z}\right\rangle & =\frac{\hbar}{2} N\left|n_{-}, n_{z}\right\rangle, \\
\hat{K}_{3}\left|n_{-}, n_{z}\right\rangle & =\frac{\hbar}{2} l\left|n_{-}, n_{z}\right\rangle,
\end{aligned}
$$

where $N=0,1,2,3, \ldots$, and $l=-N,-N+2, \ldots, N-2, N$.

5.2.1. Solving the axial-magnetron coupled Hamiltonian: the avoided crossing. Hamiltonian (36) is rewritten in terms of dressed mode operators

$$
\begin{aligned}
\hat{\mathcal{H}}_{\zeta t}\left(\epsilon_{k}\right)= & \hbar \omega_{\tau}\left(\epsilon_{k}\right)\left(\hat{n}_{\tau}+\frac{1}{2}\right) \\
& +\hbar \omega_{\mu}\left(\epsilon_{k}\right)\left(\hat{n}_{\mu}+\frac{1}{2}\right) \hbar+\tilde{\omega}_{+}\left(\hat{n}_{+}+\frac{1}{2}\right),
\end{aligned}
$$

where $\hat{n}_{\tau}$, and $\hat{n}_{\mu}$ are number operators of the magnetron and axial modes, respectively, rotated through the angle

$$
\theta_{\mathrm{m}}\left(\epsilon_{k}\right)=\arctan \left[\frac{\omega_{2}\left(\epsilon_{k}\right)}{\delta}\right]
$$

around the local $x$-axis of the basis, $\hat{K}_{1}$. These operators, along with the frequencies of the dressed modes, $\omega_{\tau}\left(\epsilon_{k}\right)$ and $\omega_{\mu}\left(\epsilon_{k}\right)$, are defined explicitly in (A.3) and (A.4).

In terms of Schwinger boson operators of the dressed modes $\tau$ and $\mu$, Hamiltonian (40) is written:

$$
\begin{aligned}
\hat{\mathcal{H}}_{\zeta t}\left(\epsilon_{k}\right)= & \omega_{0}\left(\epsilon_{k}\right) \hat{K}_{0}^{\tau \mu}+\left(\sqrt{\omega_{2}^{2}\left(\epsilon_{k}\right)+\delta^{2}}\right) \hat{K}_{3}^{\tau \mu} \\
& +\frac{\hbar \omega_{0}\left(\epsilon_{k}\right)}{2}+\hbar \tilde{\omega}_{+}\left(\epsilon_{k}\right)\left(\hat{n}_{+}+\frac{1}{2}\right),
\end{aligned}
$$


where the set $\left\{\hat{K}_{j}^{\tau \mu}\right\}$ are again obtained from $\left\{\hat{K}_{j}\right\}$ (B.9) by rotation through $\theta_{\mathrm{m}}\left(\epsilon_{k}\right)$ around $\hat{K}_{1}$. It is straightforward to interpret the effects of this 'dressing': the degeneracy of the $\hat{K}_{3}$ levels in equation (36) of the combined '-' ' and ' $z$ ' modes at the point $\delta=0$ is lifted by the non-zero value of $\omega_{2}\left(\epsilon_{k}\right)$ in equation (42). This is depicted for the $N=1$ levels in figure 3.

\subsection{Adiabaticity and the preparation of quantum states}

The sequence of elliptical driving and mode coupling in the Geonium Chip can be used to prepare the squeezed thermal state of the electron. In order that preparation is robust, each stage of the experimental process must satisfy the quantum adiabaticity theorem. First derived in 1928 by Born and Fock [44], the theorem states that, during its evolution by a Hamiltonian $\hat{H}(t)$, a quantum state prepared in an initial eigenstate $|n(0)\rangle$ remains close to the instantaneous eigenstate $|n(t)\rangle$ as time evolves [45]. A detailed calculation of the criteria for ensuring quantum adiabaticity is not included in this paper, but a general discussion is provided below.

The theorem is more easily applied to the present calculation when formulated in the parameter domain $[46,47]$ rather than the time domian. For the squeezing parts of the evolution the parameter path between an initial value $\epsilon_{\mathrm{I}}$ to a final value $\epsilon_{\mathrm{F}}$ must be followed, in principle, infinitely slowly [45]. In practice, a finite speed is possible, requiring that the voltage ratios controlling progression along this path are adjusted slowly, but over a finite time. For example, in the case of the geonium trap of section 2.1.2, if the ring voltage is held at $V_{\mathrm{r}}=-1 \mathrm{~V}$, a value of the ellipticity $\epsilon=0.999941$ requires a voltage of the side-electrodes $V_{\mathrm{g}}=-1.83 \mathrm{~V}$, with a possible correction-electrode voltage of $V_{c}^{\text {opt }}=-1.14 \mathrm{~V}$ to ensure the trap remains harmonic [25]. The voltages $V_{\mathrm{g}}$ and $V_{\mathrm{c}}$ must be driven to these values in piecewise steps, as discussed, with the voltages at each increment used to calculate the resulting ellipticity of the trap, and via the Hamiltonian (27), the initial and final energy of the system. The speed of the ramping process at each step will be determined by the relative size of the gap in the energy spectrum, $\Delta E$, to the timescale over which the voltages are changed, $\tau$, such that $\tau \gg \hbar / \Delta E$.

For the mode coupling stages of the process, the problem can be described by analogy with the Landau-Zener (LZ) model $[48,49]$. Figure 3 shows the splitting $\hbar \omega_{2}(\epsilon)$ between the energy levels due to the dressing of the trap potentials by the external field. Only the lowest split energy levels of the combined axial and magnetron modes are included. Although a simplified representation of the spectrum, it illustrates the adiabaticity condition as applied to this process. During the coupling stages, the ellipticity is assumed to be held at a constant value. Referring to figure 3 , the system will remain in an adiabatic state (red line) by following either the path indicated by the blue or green arrows as the gap at $\delta=0$ is created when the coupling field in (35) is first switched on. Avoiding transition between the adiabatic states requires that the time associated with such a transition $\rightarrow \infty$. This can be satisfied by achieving a large enough gap $\hbar \omega_{2}(\epsilon)$, as provided by the field strength $\xi$ in (34).

\section{Summary}

We have discussed the general quantum theory of the elliptical Penning trap in this paper, and have presented the explicit relationship between the variation of the trapping potential, and the squeezing of the normal modes of the system. In combining this theory with the tunable mechanism of the Geonium Chip, we have shown how the trap can be used to prepare these states with a high degree of control. The quantum theory of mode coupling was reviewed and discussed in the context of the combined energy level spectrum of the axial and magnetron modes, which enables a dressed-atom approach to the solution. This led to a discussion of the adiabatic preparation of the system, prompting future theoretical work into a full derivation of the adiabaticity constraints. The work highlights the range of operations which can be performed using the motional degrees of freedom of an electron in the Geonium Chip, and in other Penning traps with tunable ellipticity, promoting these traps as candidates in experimental quantum optics.

\section{Acknowledgments}

This work was supported by the UK Engineering and Physical Sciences Research Council [Grant No. EP/K503198/1].

\section{Data availability statement}

No new data were created or analysed in this study.

\section{Appendix A. Useful definitions}

$$
\begin{gathered}
\theta\left(\epsilon_{k}, \delta \epsilon_{k}\right)=\arctan \left[\frac{\delta \epsilon_{k} \omega_{z}^{2}}{\tilde{\omega}_{1}\left(\epsilon_{k}\right) \tilde{\omega}_{c}\left(\epsilon_{k}\right)}\right] \\
\tilde{\omega}_{\mathrm{c}}\left(\epsilon_{k}\right)=\tilde{\omega}_{+}\left(\epsilon_{k}\right)+\tilde{\omega}_{-}\left(\epsilon_{k}\right), \quad \tilde{\omega}_{1}\left(\epsilon_{k}\right)=\tilde{\omega}_{+}\left(\epsilon_{k}\right)-\tilde{\omega}_{-}\left(\epsilon_{k}\right) \\
\hat{n}_{\tau}=\hat{a}_{\tau}^{\dagger}\left(\epsilon_{k}\right) \hat{a}_{\tau}\left(\epsilon_{k}\right), \quad \hat{n}_{\mu}=\hat{a}_{\mu}^{\dagger}\left(\epsilon_{k}\right) \hat{a}_{\mu}\left(\epsilon_{k}\right) ; \\
\hat{a}_{\tau}^{\dagger}\left(\epsilon_{k}\right)=\cos \frac{\theta_{m}\left(\epsilon_{k}\right)}{2} \hat{a}_{-}^{\dagger}-\mathrm{i} \sin \frac{\theta_{m}\left(\epsilon_{k}\right)}{2} \hat{a}_{z}^{\dagger} \\
\hat{a}_{\tau}\left(\epsilon_{k}\right)=\cos \frac{\theta_{m}\left(\epsilon_{k}\right)}{2} \hat{a}_{-}+\mathrm{i} \sin \frac{\theta_{m}\left(\epsilon_{k}\right)}{2} \hat{a}_{z} \\
\hat{a}_{\mu}^{\dagger}\left(\epsilon_{k}\right)=\cos \frac{\theta_{m}\left(\epsilon_{k}\right)}{2} \hat{a}_{z}^{\dagger}-\mathrm{i} \sin \frac{\theta_{m}\left(\epsilon_{k}\right)}{2} \hat{a}_{-}^{\dagger} \\
\hat{a}_{\mu}\left(\epsilon_{k}\right)=\cos \frac{\theta_{m}\left(\epsilon_{k}\right)}{2} \hat{a}_{z}+\mathrm{i} \sin \frac{\theta_{m}\left(\epsilon_{k}\right)}{2} \hat{a}_{-} \\
\omega_{\tau}\left(\epsilon_{k}\right)=\frac{1}{2}\left(\omega_{0}\left(\epsilon_{k}\right)+\sqrt{\omega_{2}^{2}\left(\epsilon_{k}\right)+\delta^{2}}\right) \\
\omega_{\mu}\left(\epsilon_{k}\right)=\frac{1}{2}\left(\omega_{0}\left(\epsilon_{k}\right)-\sqrt{\omega_{2}^{2}\left(\epsilon_{k}\right)+\delta^{2}}\right)
\end{gathered}
$$




\section{Appendix B. Sideband-coupling}

The coupling potential (35) is quantized, with the $\hat{x}$ and $\hat{z}$ operators written as combinations of the $z,+$ and - operators in (16) and (17):

$$
\begin{aligned}
\hat{V}\left(\hat{x}, \hat{z}, \epsilon_{k}, t\right)= & -\varepsilon_{m} \cos \left(\tilde{\omega}_{m}\left(\epsilon_{k}\right) t\right) \hat{x} \hat{z} \\
= & -\frac{\hbar}{2 m} \frac{1}{\sqrt{\omega_{1} \omega_{z}}} \xi \cos \left(\tilde{\omega}_{m}\left(\epsilon_{k}\right) t\right) \\
& \times\left\{\left(\hat{a}_{z}+\hat{a}_{z}^{\dagger}\right)\left(\hat{a}_{+}+\hat{a}_{+}^{\dagger}+\hat{a}_{-}+\hat{a}_{-}^{\dagger}\right)\right\} .
\end{aligned}
$$

This is first transformed to the frame in which the elliptical Hamiltonian is diagonal, namely by transforming by $\hat{U}$, $\hat{S}\left(\zeta_{+}\left(\epsilon_{k}\right)\right)$ and $\hat{S}\left(\zeta_{-}\left(\epsilon_{k}\right)\right)$ in succession, resulting in

$$
\begin{aligned}
\hat{V}_{\zeta}\left(\hat{x}, \hat{z}, \epsilon_{k}, t\right)= & \hat{S}\left(\zeta_{+}\left(\epsilon_{k}\right)\right) \hat{S}\left(\zeta_{-}\left(\epsilon_{k}\right)\right) \hat{U} \hat{\tilde{V}}\left(\epsilon_{k}, t\right) \\
& \times \hat{U}^{\dagger} \hat{S}^{\dagger}\left(\zeta_{-}\left(\epsilon_{k}\right)\right) \hat{S}^{\dagger}\left(\zeta_{+}\left(\epsilon_{k}\right)\right) \\
= & -\xi \cos \left(\tilde{\omega}_{m}\left(\epsilon_{k}\right) t\right) \frac{\hbar}{2 m} \frac{1}{\sqrt{\omega_{1} \omega_{z}}} \\
& \times\left\{( \hat { a } _ { z } + \hat { a } _ { z } ^ { \dagger } ) \left(A_{+}\left(\theta, \zeta_{+}\right) \hat{a}_{+}+A_{+}^{\dagger}\left(\theta, \zeta_{+}\right) \hat{a}_{+}^{\dagger}\right.\right. \\
& \left.\left.+A_{-}^{\dagger}\left(\theta, \zeta_{-}\right) \hat{a}_{-}+A_{-}^{\dagger}\left(\theta, \zeta_{-}\right) \hat{a}_{-}\right)\right\},
\end{aligned}
$$

where the amplitudes $A_{ \pm}^{(\dagger)}\left(\theta, \zeta_{ \pm}\right)$are given by

$$
\begin{aligned}
& A_{ \pm}\left(\theta, \zeta_{ \pm}\right)=\cosh \left(\zeta_{ \pm}\right) \exp (\mathrm{i} \theta / 2)+\sinh \left(\zeta_{ \pm}\right) \exp (-\mathrm{i} \theta / 2) \\
& A_{ \pm}^{\dagger}\left(\theta, \zeta_{ \pm}\right)=\cosh \left(\zeta_{ \pm}\right) \exp (-\mathrm{i} \theta / 2)+\sinh \left(\zeta_{ \pm}\right) \exp (\mathrm{i} \theta / 2)
\end{aligned}
$$

The total Hamiltonian is now

$$
\hat{\mathcal{H}}_{\zeta}^{\prime}\left(\epsilon_{k}\right)=\hat{\mathcal{H}}_{\zeta}+q \hat{V}_{\zeta}\left(\epsilon_{k}, t\right)
$$

The explicit time dependence of the additional potential $\hat{V}_{\zeta}$ can be removed by transforming Hamiltonian (B.4) by the unitary operator

$$
\hat{U}_{m}(t)=\exp \left\{-\frac{\mathrm{i}}{\hbar}\left(\tilde{\omega}_{m}\left(\epsilon_{k}\right) t\right) \hat{K}_{3}\right\},
$$

where

$$
\hat{K}_{3}=\frac{\hbar}{2}\left(\hat{n}_{-}-\hat{n}_{z}\right)
$$

Expanding out the cosine function in the applied potential leads to two stationary terms in $q \hat{V}_{\zeta}$, with the others rotating at $\mathrm{MHz}$ frequencies. A secular approximation [50], and the choice of coupling frequency

$$
\tilde{\omega}_{m}\left(\epsilon_{k}\right)=\tilde{\omega}_{-}\left(\epsilon_{k}\right)+\omega_{z}+\delta
$$

results in the total Hamiltonian:

$$
\begin{aligned}
\hat{\mathcal{H}}_{\zeta t}^{\prime}\left(\epsilon_{k}\right)= & \hat{U}_{m}(t) \hat{\mathcal{H}}_{\zeta}^{\prime}\left(\epsilon_{k}\right) \hat{U}_{m}^{\dagger}(t)+\mathrm{i} \hbar \hat{\dot{U}}_{m}(t) \hat{U}_{m}^{\dagger}(t) \\
= & \omega_{0}\left(\epsilon_{k}\right) \hat{K}_{0}+\delta \hat{K}_{3}-\omega_{2}\left(\epsilon_{k}\right) \hat{K}_{2}+\omega_{1}\left(\epsilon_{k}\right) \hat{K}_{1} \\
& +\frac{\hbar \omega_{0}\left(\epsilon_{k}\right)}{2}+\hbar \omega_{+}\left(\hat{n}_{+}+\frac{1}{2}\right),
\end{aligned}
$$

where

$$
\begin{aligned}
\hat{K}_{0} & =\frac{\hbar}{2}\left(\hat{n}_{-}+\hat{n}_{z}\right), \\
\hat{K}_{1} & =\frac{\hbar}{2}\left(\hat{a}_{-}^{\dagger} \hat{a}_{z}+\hat{a}_{z}^{\dagger} \hat{a}_{-}\right), \\
\hat{K}_{2} & =-\frac{\mathrm{i} \hbar}{2}\left(\hat{a}_{-}^{\dagger} \hat{a}_{z}-\hat{a}_{z}^{\dagger} \hat{a}_{-}\right),
\end{aligned}
$$

and

$$
\begin{aligned}
& \omega_{0}\left(\epsilon_{k}\right)=\omega_{z}-\tilde{\omega}-\left(\epsilon_{k}\right), \\
& \omega_{2}\left(\epsilon_{k}\right)=\frac{e}{2 m} \frac{1}{\sqrt{\omega_{1} \omega_{z}}} \sin \frac{\theta\left(\epsilon_{k}\right)}{2} \exp \left(-\zeta_{-}\left(\epsilon_{k}\right)\right) \xi, \\
& \omega_{1}\left(\epsilon_{k}\right)=\frac{e}{2 m} \frac{1}{\sqrt{\omega_{1} \omega_{z}}} \cos \frac{\theta\left(\epsilon_{k}\right)}{2} \exp \left(\zeta_{-}\left(\epsilon_{k}\right)\right) \xi
\end{aligned}
$$

Referring to figure 3, it is clear that for $\epsilon_{k}>0, \zeta_{-}<0$, and so $\exp \left(\zeta_{-}\left(\epsilon_{k}\right)\right)<1$ in $\omega_{1}\left(\epsilon_{k}\right)$ (B.10). It follows that the strength of the $\hat{K}_{1}$ term in (B.8) becomes vanishingly small as the electron is driven to the ultra-elliptical regime. The coupling strength in front of $\hat{K}_{2}$ however, will become increasingly strong as $\epsilon_{k} \rightarrow 1$. Indeed, it is as this regime is approached that the magnetron frequency decreases, and hence the magnetron quantum number increases, most rapidly [25], corresponding to the point at which on-resonance magnetron cooling becomes crucial. We therefore neglect the $\hat{K}_{1}$ contribution in the Hamiltonian (36), and proceed to define

$$
\begin{aligned}
\hat{\mathcal{H}}_{\zeta t}= & \omega_{0}\left(\epsilon_{k}\right) \hat{K}_{0}+\delta \hat{K}_{3}-\omega_{2}\left(\epsilon_{k}\right) \hat{K}_{2} \\
& +\frac{\hbar \omega_{0}\left(\epsilon_{k}\right)}{2}+\hbar \tilde{\omega}_{+}\left(\hat{n}_{+}+\frac{1}{2}\right) .
\end{aligned}
$$

\section{ORCID iDs}

Frances Crimin (D) https://orcid.org/0000-0002-7873-3525 José L Verdú (D) https://orcid.org/0000-0001-5460-3040

\section{References}

[1] Dehmelt H 1990 Experiments with an isolated subatomic particle at rest 44th Annual Symp. on Frequency Control pp 525-31

[2] Blaum K 2006 High-accuracy mass spectrometry with stored ions Phys. Rep. $4251-78$

[3] Sturm S, Köhler F, Zatorski J, Wagner A, Harman Z, Werth G, Quint W, Keitel C H and Blaum K 2014 High-precision measurement of the atomic mass of the electron Nature $\mathbf{5 0 6}$ 467-70

[4] Hanneke D, Fogwell S and Gabrielse G 2008 New measurement of the electron magnetic moment and the fine structure constant Phys. Rev. Lett. 100120801 
[5] Rodegheri C C, Blaum K, Kracke H, Kreim S, Mooser A, Quint W, Ulmer S and Walz J 2012 An experiment for the direct determination of theg-factor of a single proton in a Penning trap New J. Phys. 14063011

[6] Mooser A et al 2014 Direct high-precision measurement of the magnetic moment of the proton Nature 509 596-9

[7] Ulmer S et al 2005 Penning trap measurement of the magnetic moment of the antiproton AIP Conf. Proc., LEAP 796 260-5

[8] Gabrielse $\mathrm{G}$ et al 2012 Trapped antihydrogen in its ground state Phys. Rev. Lett. 108113002

[9] Yamazaki Y 2012 Antimatter matters: progress in cold antihydrogen Research J. Phys.: Conf. Ser. 388012002

[10] Smorra C et al 2017 A parts-per-billion measurement of the antiproton magnetic moment Nature $550371-4$

[11] Marzoli I et al 2009 Experimental and theoretical challenges for the trapped electron quantum computer J. Phys. B: At. Mol. Opt. Phys. 42154010

[12] Ciaramicoli G, Marzoli I and Tombesi P 2003 Scalable quantum processor with trapped electrons Phys. Rev. Lett. 91017901

[13] Pedersen L H and Rangan C 2008 Controllability and universal three-qubit quantum computation with trapped electron states Quantum Inf. Process. 7 33-42

[14] Lamata L, Porras D, Cirac J I, Goldman J and Gabrielse G 2010 Towards electron-electron entanglement in Penning traps Phys. Rev. A 81022301

[15] Ciaramicoli G, Marzoli I and Tombesi P 2001 Realization of a quantum algorithm using a trapped electron Phys. Rev. A 63 052307

[16] Stahl S, Galve F, Alonso J, Djekic S, Quint W, Valenzuela T, Verdú J, Vogel M and Werth G 2005 A planar Penning trap Eur. Phys. J. D 32 139-46

[17] Castrejón-Pita J R and Thompson R C 2005 Proposal for a planar Penning ion trap Phys. Rev. A 72013405

[18] Hellwig M, Bautista-Salvador A, Singer K, Werth G and Schmidt-Kaler F 2010 Fabrication of a planar micro Penning trap and numerical investigations of versatile ion positioning protocols New J. Phys. 12065019

[19] Goldman J and Gabrielse G 2010 Optimized planar Penning traps for quantum-information studies Phys. Rev. A 81 052335

[20] Jain S, Alonso J, Grau M and Home J P 2020 Scalable arrays of micro-penning traps for quantum computing and simulation Phys. Rev. X 10031027

[21] Ciaramicoli G, Marzoli I and Tombesi P 2010 From a single- to a double-well Penning trap Phys. Rev. A 82044302

[22] Verdú J 2011 Theory of the coplanar-waveguide Penning trap New J. Phys. 13113029

[23] Verdú J 2013 Patent, Ion Trap, US 8362423 B1

[24] Verdú J 2013 Patent, Ion Trap, WO 2013/041615 A2

[25] Pinder J and Verdú J 2013 A planar Penning trap with tunable dimensionality of the trapping potential Int. J. Mass Spectrom. 356 49-59

[26] Pinder J 2016 The Geonium Chip: engineering a scalable planar Penning trap PhD Thesis University of Sussex

[27] Cridland A, Lacy J, Pinder J and Verdú J 2016 Single microwave photon detection with a trapped electron Photonics 3 59-73

[28] Kretzschmar M 2008 Theory of the elliptical Penning trap Int. J. Mass Spectrom. 275 21-33
[29] Brown L S and Gabrielse G 1986 Geonium theory: physics of a single electron or ion in a Penning trap Rev. Mod. Phys. 58 233-311

[30] Breitenfeldt M, Baruah S, Blaum K, Herlert A, Kretzschmar M, Martinez F, Marx G, Schweikhard L and Walsh N 2008 The elliptical Penning trap: experimental investigations and simulations Int. J. Mass Spectrom. 275 34-44

[31] Crimin F 2017 Quantum theory of the penning trap PhD Thesis University of Sussex

[32] Peil S and Gabrielse G 1999 Observing the quantum limit of an electron cyclotron: QND measurements of quantum jumps between Fock states Phys. Rev. Lett. 83 1287-90

[33] Goodwin J F, Stutter G, Thompson R C and Segal D M 2016 Resolved-sideband laser cooling in a penning trap Phys. Rev. Lett. 116143002

[34] Landau L D and Lifshitz E M 1975 The Classical Theory of Fields: Volume 2 (Course of Theoretical Physics) (Oxford: Pergamon)

[35] Kretzschmar M 1991 Particle motion in a Penning trap Eur. J. Phys. 12 240-6

[36] Wineland D and Dehmelt H 1975 Line shifts and widths of axial, cyclotron and G-2 resonances in tailored, stored electron (ion) cloud Int. J. Mass Spectrom. Ion Phys. 16 $338-42$

[37] Gabrielse G and Mackintosh F C 1984 Cylindrical Penning traps with orthogonalized anharmonicity compensation Int. J. Mass Spectrom. Ion Process. 57 1-17

[38] Barnett S and Radmore P 2002 Methods in Theoretical Quantum Optics (New York: Oxford University Press)

[39] Kretzschmar M 1992 Single particle motion in a Penning trap: description in the classical canonical formalism Phys. Scr. $\mathbf{4 6}$ $544-54$

[40] Crimin F, Garraway B M and Verdú J 2018 The quantum theory of the Penning trap J. Mod. Opt. $\mathbf{6 5} 427-40$

[41] Wang C-C J, Keith A C and Freericks J K 2013 Phononmediated quantum spin simulator employing a planar ionic crystal in a Penning trap Phys. Rev. A 8713422

[42] Cornell E A, Weisskoff R M, Boyce K R and Pritchard D E 1990 Mode coupling in a Penning trap: $\pi$ pulses and a classical avoided crossing $41312-5$

[43] Schwinger J 1965 On Angular Momentum (New York: Dover )

[44] Born M and Fock V 1928 Beweis des Adiabatensatzes Z. Physik 51 165-80

[45] Comparat D 2009 General conditions for quantum adiabatic evolution Phys. Rev. A 80012106

[46] Wu Z and Yang H 2005 Validity of the quantum adiabatic theorem Phys. Rev. A 72012114

[47] Liu J and Fu L-B 2007 Singularities of Berry connections inhibit the accuracy of the adiabatic approximation Phys. Lett. A $\mathbf{3 7 0}$ $17-21$

[48] Landau L 1965 Theory of energy transfer II Collected Papers of L.D. Landau D Ter Harr (Oxford: Pergamon)

[49] Zener C 1932 Non-adiabatic crossing of energy levels Proc. $R$. Soc. A 137 696-702

[50] Cohen-Tannoudji C and Reynaud S 1977 Dressed-atom description of resonance fluorescence and absorption spectra of a multi-level atom in an intense laser beam J. Phys. B: At. Mol. Phys. 10 345-63 